\title{
Efecto de los factores climáticos, variedades y densidades de siembra en la dinámica de artrópodos en cultivos de arroz en Yopal-Casanare, Colombia
}

\author{
Effect of climatic factors, plant varieties, and planting densities on arthropod dynamics in \\ rice crops in Yopal-Casanare, Colombia
}

\author{
DIANA OBREGÓN-CORREDOR ${ }^{1}$; FRANCISCO JAVIER \\ HERNÁNDEZ-GUZMÁN ${ }^{2}$; DIANA KATHERINNE RIOS-MOYANO ${ }^{3}$
}

\begin{abstract}
${ }^{1}$ M. Sc. Agrarias. Estudiante de Doctorado, Departamento de Entomología, Universidad de Cornell, Ithaca, Nueva York, Estados Unidos, do265@cornell.edu, https://orcid.org/0000-0002-6582-9149. ${ }^{2}$ Ingeniero Agrónomo. Estudiante de M.Sc Meteorología, Universidad Nacional de Colombia, Federación Nacional de Arroceros - FEDEARROZ, Yopal, Colombia, franciscojavierhernandez@fedearroz.com.co, https://orcid.org/0000-0002-5074-5188. ${ }^{3}$ M. Sc. Biológicas. Docente tiempo Completo, Facultad de Ciencias Agropecuarias, Programa de Ingeniería Agronómica, Universidad de La Salle, Yopal, Colombia, dkrios@unisalle.edu.co, https://orcid.org/0000-0002-5917-9100.
\end{abstract}

\begin{abstract}
Autor para correspondencia
Diana Obregón Corredor. M. Sc. Agrarias. Estudiante de Doctorado, Departamento de Entomología, Universidad de Cornell, Ithaca, Nueva York, Estados Unidos,do265@cornell. $e d u$, https://orcid.org/0000-0002-6582-9149.
\end{abstract}

\section{Citación sugerida}

OBREGÓN-CORREDOR, D.; HERNÁNDEZ-GUZMÁN, F. J.; RIOS-MOYANO, D. K 2021. Efecto de los factores climáticos, variedades y densidades de siembra en la dinámica de artrópodos en cultivos de arroz en Yopal-Casanare, Colombia. Revista Colombiana de Entomología 47 (1): e9364. https://doi.org/10.25100/ socolen.v47i1.9364

Recibido: 02-abr-2019

Aceptado: 11-sep-2020

Publicado: 15-jun-2021

Revista Colombiana de Entomología ISSN (Print): 0120-0488

ISSN (On Line): 2665-4385

https://revistacolombianaentomologia.univalle.edu.co

Open access

(c) (i) (2) BY-NC-SA 4.0

Publishers: Sociedad Colombiana de Entomología SOCOLEN (Bogotá, D. C., Colombia) https://www.socolen.org.co

Universidad del Valle (Cali, Colombia)

https://www.univalle.edu.co

(C) 2021 Sociedad Colombiana de Entomología SOCOLEN y Universidad del Valle - Univalle
Resumen: Se evaluó la diversidad y dinámica poblacional de la artropofauna presente en cultivos de arroz bajo los sistemas de siembra secano y riego, con dos variedades de arroz (Fedearroz 174 y Fedearroz 733 ) y en tres densidades de siembra $(60,100$ y $180 \mathrm{~kg} / \mathrm{ha})$ en relación a diferentes variables climáticas. Para la recolección de especímenes, se realizaron 10 pases dobles de red entomológica cada ocho días desde la emergencia hasta el inicio de la fase de maduración del grano. Los morfotipos encontrados fueron identificados y agrupados según su tipo de interacción con el cultivo en chupadores, masticadores, depredadores y parasitoides. Las variables climáticas más relevantes se seleccionaron con un análisis de Random Forest. Se identificaron 126 morfotipos en 12 órdenes. La mayor riqueza se encontró en Hemiptera, Coleoptera y Diptera, así como en el grupo de chupadores. No se encontraron diferencias en la abundancia o riqueza de artrópodos entre variedades o densidades de siembra. El sistema de siembra con riego presentó una mayor abundancia total de artrópodos en comparación con el sistema secano, pero en este sistema hubo una mayor riqueza de especies. Las poblaciones de parasitoides y depredadores se correlacionaron negativamente con los masticadores, pero no se presentó ningún efecto sobre los chupadores. La temperatura y la humedad relativa fueron las variables climáticas de mayor relevancia en la dinámica de los artrópodos. Estos resultados contribuyen para el desarrollo de sistemas de monitoreo y manejo de plagas que tengan en cuenta las dinámicas de interacción entre grupos ecológicos y el efecto del clima local.

Palabras clave: Dinámica poblacional, diversidad funcional, temperatura, humedad relativa, artrópodos, Tagosodes orizicolus.

Abstract: The diversity and population dynamics of the arthropods present in rice crops under dry and irrigated planting systems were evaluated, with two rice varieties (Fedearroz 174 and Fedearroz 733), and in three planting densities $(60,100$, and $180 \mathrm{~kg} / \mathrm{ha})$ in relation to different climatic variables. For specimen collection, 10 double entomological net passes were performed every eight days from emergence to the start of the maturation phase. The morphotypes found were identified and grouped according to their type of interaction with the crop in sap-suckers, chewers, predators, and parasitoids. The most relevant climatic variables were selected with a Random Forest analysis. 126 morphotypes were identified in 12 orders. The greatest richness was found in Hemiptera, Coleoptera and Diptera, as well as in the group of suckers. No differences were found in the abundance or richness of arthropods between varieties or planting densities. The irrigation system presented a greater total abundance of arthropods compared to the rainfed system, but in this system, there was higher species richness. Parasitoid and predator populations were negatively correlated with chewers but there was no effect on sap-suckers. Temperature and relative humidity were the most relevant climatic variables in arthropod dynamics. These results contribute to develop pest monitoring and management systems that take into account the dynamics of interaction between ecological groups and the effect of local climate.

Keywords: Population dynamic, functional diversity, temperature, relative humidity, arthropods, Tagosodes orizicolus.

\section{Introducción}

Colombia produjo alrededor de tres millones de toneladas de arroz durante 2019 en 539.553 hectáreas, de las cuales 159.082 fueron cultivadas en el departamento de Casanare (Departamento Administrativo Nacional de Estadística 2019; 2020). 
El $80 \%$ de esta área se cultiva en el primer semestre del año en el sistema de siembra secano, el cual depende por completo del agua lluvia, que inicia el ciclo entre abril y mayo. El $20 \%$ restante se cultiva con el sistema de siembra con riego, donde se requiere agua para irrigar durante todo el ciclo de cultivo dado que es la temporada seca, iniciando de agosto a noviembre (Federación Nacional de Arroceros 2011). Por el manejo en sí y por los cambios en el clima a lo largo del año, estos dos sistemas de cultivo generan una diversidad de condiciones para los artrópodos impactando la abundancia de las poblaciones.

La clasificación de la fauna asociada a un agroecosistema según su relación trófica (herbívoro, depredador, parasitoide, etc.) ha demostrado ser útil para entender de forma general la estructura y dinámica de interacción de las poblaciones (Settle et al. 1996). En las plantaciones de arroz se han reportado diferentes insectos herbívoros considerados plagas tales como Tagosodes orizicolus Muir, 1926 (Hemiptera: Delphacidae) conocido como la sogata del arroz, minadores de la hoja del género Hydrellia Cresson, 1932 (Diptera: Ephydridae), diferentes especies de larvas como Spodoptera frugiperda Smith, 1791 (Lepidoptera: Noctuidae), Diatraea sp. (Lepidoptera: Crambidae), Rupela albinella Stoll, 1781(Lepidoptera: Pyralidae), Mocis latipes Gueneé, 1852 (Lepidoptera: Erebidae), e insectos chupadores que afectan el llenado del grano como Euschistus sp. (Hemiptera: Pentatomidae), Oebalus insularis Stål, 1872 (Hemiptera: Pentatomidae), Tibraca limbativentris Stål, 1860 (Hemiptera: Pentatomidae) y Blissus Burmeister, 1835 (Hemiptera: Blissidae) (Pérez y Cuevas 2012; Saavedra de Castro y Fernández Herrera 2019). Estos insectos plaga causan del 10 al $12 \%$ de pérdidas en el rendimiento y alrededor de $17 \%$ de los gastos en medidas de control (Vivas y Notz 2011; Federación Nacional de Arroceros 2019). Sin embargo, esa no es la única fauna atrópoda presente en el cultivo, existe una diversidad de grupos de enemigos naturales tales como arañas (He et al. 2020), avispas o chinches depredadores que juegan un rol importante en la regulación de plagas (Meneses 2008; Lou et al. 2013).

Las poblaciones de insectos en los cultivos son afectadas por factores como el clima, las prácticas agronómicas y los ciclos de cultivo (Welch y Harwood 2014). Teniendo en cuenta estos factores y con el fin de aportar información para el desarrollo de estrategias de monitoreo, sistemas de alerta temprana y manejo integrado de plagas (Reyna et al. 1994; Costa et al. 2018) en los nuevos escenarios dados por el cambio climático (Lamichhane et al. 2015), el propósito de este trabajo fue caracterizar la artropofauna presente en cultivos de arroz en las condiciones de Yopal, Casanare, junto con una aproximación a la dinámica de estas poblaciones a lo largo de los diferentes ciclos del cultivo en relación con las variables climáticas. Esto además, en dos variedades de arroz comúnmente usadas en la región y tres densidades de siembra.

\section{Materiales y métodos}

El estudio se realizó en la zona rural del municipio de Yopal, Casanare, Colombia, en las instalaciones de la Universidad de La Salle, finca Matepantano ( $5^{\circ} 19^{\prime} 30^{\prime \prime} \mathrm{N}$ y $\left.72^{\circ} 18^{\prime} 2^{\prime} \mathrm{O}\right)$, en un área tradicionalmente arrocera. Ubicada a 240 metros sobre el nivel del mar, con temperatura promedio de $24{ }^{\circ} \mathrm{C}$ y precipitación de $2.500 \mathrm{~mm}$ anuales, con un comportamiento monomodal alcanzando su máxima precipitación en mayo con más de $350 \mathrm{~mm}$ (Bustamante Lozano et al. 2013).
Se sembraron dos variedades de semillas comerciales de arroz: Fedearroz 174 y Fedearroz 733, bajo tres densidades de siembra: $60 \mathrm{~kg} /$ ha densidad baja para la zona, $100 \mathrm{~kg} / \mathrm{ha}$ densidad recomendada por Fedearroz y $180 \mathrm{~kg} / \mathrm{ha}$ densidad de siembra promedio que se utiliza en la zona, con parcelas de $100 \mathrm{~m}^{2}$ con cuatro repeticiones en un diseño de bloques completos al azar. Se garantizó la uniformidad del terreno con el fin de evitar concentraciones de humedad que dieran condiciones favorables a algún tipo de insecto y no se realizó ningún tipo de aplicación de insecticidas, salvo el tratamiento inicial que acompañaba la semilla compuesto de imidacloprid (233 g/l) a razón de $3 \mathrm{ml} / \mathrm{kg}$ de semilla. La fertilización se realizó con base en el análisis de suelos en cinco fraccionamientos, un pre-abono y cuatro aplicaciones en post-emergencia, con las siguientes unidades totales aplicadas: nitrógeno 151,5, fósforo $60 \mathrm{y}$ potasio 150 .

Los muestreos de artrópodos se efectuaron cada ocho días realizando 10 pases dobles de jama (10 PDJ), a partir de los 8 días después de germinación, durante las fases vegetativa y reproductiva y hasta el inicio de la etapa de llenado del grano entre los 92 y 96 días después de germinación. Estos muestreos se realizaron para dos ciclos de cultivo, el primero en el sistema de siembra secano durante junio, julio y agosto de 2013 y el segundo en el sistema de siembra riego durante diciembre de 2013, enero y febrero de 2014.

Las muestras fueron llevadas al laboratorio de entomología de la Universidad de La Salle, donde se separaron los especímenes y se hizo conteo e identificación mediante claves taxonómicas (Triplehorn y Jhonson 2005; Fernández y Sharky 2006; Fernández et al. 2007; Rengifo-Correa y González 2011). Posteriormente, con base en la identificación y revisión de literatura sobre su biología y comportamiento en el cultivo, se le asignó a cada morfotipo una de las siguientes categorías de grupo funcional dentro del agroecosistema: depredador, parasitoide, chupador de follaje, masticador de follaje, minador o desconocido para entender las relaciones tróficas y las dinámicas a lo largo del ciclo del cultivo (Ríos Romero 2003; Bedoya et al. 2018; León-Burgos et al. 2019).

Se realizó un análisis descriptivo de la riqueza y abundancia de artrópodos encontrados y se usaron modelos lineales de efectos mixtos para comparar las variedades, las densidades de siembra y los sistemas de siembra usando como efecto aleatorio la parcela con la función lmer del paquete lme4 en $\mathrm{R}$ (R Core Team 2019). El mismo tipo de análisis fue usado para evaluar la interacción en las abundancias entre grupos funcionales.

Durante los mismos periodos de muestreo se registraron las temperaturas mínimas $\left({ }^{\circ} \mathrm{C}\right)$, máximas $\left({ }^{\circ} \mathrm{C}\right)$ y promedio $\left({ }^{\circ} \mathrm{C}\right)$, humedad relativa $(\%)$, precipitación $(\mathrm{mm})$ y radiación solar $\left(\mathrm{Cal} / \mathrm{cm}^{2} /\right.$ día) con una estación metereológica Davis Vantage Pro 2 y se utilizó el software Water link 6.0 de apoyo para la captura de los datos. Posteriormente, se determinó la relevancia de cada variable climática en relación con los cambios en abundancia de las especies encontradas utilizando el modelo Random Forest (Breiman 2001). Este análisis se llevó a cabo usando los datos climáticos promedio de los ocho días transcurridos entre muestreo y muestreo, en relación con el promedio de artrópodos encontrados por tratamiento para cada muestreo. Para esto se empleó el paquete Random Forest en $\mathrm{R}$ ( $\mathrm{R}$ Core Team 2019), generando 2.000 árboles y 100 bosques para cada análisis. Como resultado se presenta el porcentaje de árboles y bosques en los que cada variable climática fue la más discriminante para cada grupo de artrópodos. 
Además, se generaron gráficas de las relaciones parciales entre las variables climáticas más relevantes y las abundancias de los grupos tróficos y las familias de artrópodos más abundantes, detallando los puntos de inflexión en las poblaciones usando la funcion partialplot del mismo paquete en R.

\section{Resultados y discusión}

Diversidad encontrada. Se recolectaron 16.768 especímenes, dentro de los cuales se identificaron 126 morfotipos en 12 órdenes. 106 morfotipos fueron agrupados en 53 familias y 42 morfotipos a nivel de género o especie. Hemiptera, Coleoptera, Diptera, Hymenoptera y Lepidoptera fueron los órdenes con más riqueza de especies (Fig. 1). Mientras que Hemiptera, Diptera, Araneae, Lepidoptera, Hymenoptera, Coleoptera y Orthoptera los de mayor abundancia (Fig. 2).

En los grupos funcionales se encontró que las especies de hábito chupador, así como los masticadores de follaje son los grupos más diversos, que junto con los minadores de hojas completan el $50 \%$ de la diversidad de especies en el cultivo con hábito herbívoro (Fig. 3). En cuanto a la abundancia, los depredadores fueron el grupo más abundante, seguido de los masticadores y chupadores de follaje (Fig. 4).

Dentro de los depredadores se encontró 20 morfotipos de los cuales el $50 \%$ fueron arañas Tetragnathidae (Araneae), con una especie del género Tetragnatha sp. como la más abundante de las recolectas (Tabla 1). En otros estudios ya se ha reconocido este género como depredadores muy efectivos para el control de T. orizicolus reduciendo sus poblaciones entre un 25 y $68 \%$ cuando hay una densidad de cuatro Tetragnatha por metro cuadrado, siendo capaz de alimentarse hasta 3,5 adultos de T. orizicolus por día (Cuevas 1994). En este grupo también se encontraron libélulas Coenagrionidae, adultos de Syrphidae y avispas Vespidae y Pompilidae.

En el grupo de los chupadores se presentó un complejo de 32 morfotipos del orden Hemiptera, dentro del que se encuentran T. orizicolus, Draeculacephala Ball, 1902 (Hemiptera: Cicadellidae), Oebalus, Euschistus, Blissus, y Aeneolamiavaria Fabricius, 1781 (Hemiptera: Cercopidae), conocidos tradicio nalmente como plagas del arroz (Meneses 2008). T. orizicolus en particular, se destaca como el segundo morfotipo más abundante durante todo el estudio con 2.078 individuos encontrados (Tabla 1). T. orizicolus es reportada localmente

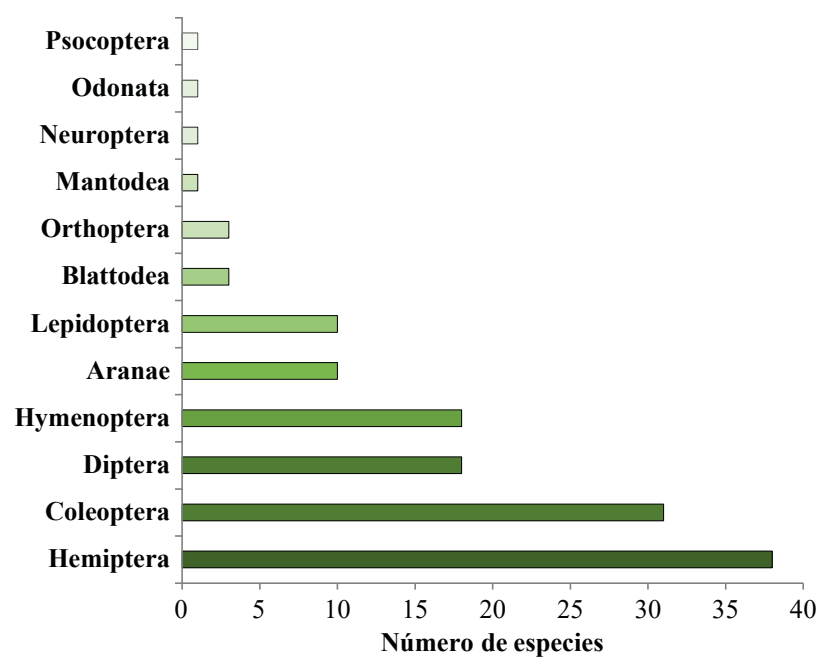

Figura 1. Riqueza de especies según el orden en cultivos de arroz en Yopal, Casanare. como una de las principales plagas del arroz (Peñaranda Rincón et al. 1999), no solo por su hábito chupador, sino por ser vector el virus de la hoja blanca que genera pérdidas para el sector (Meneses et al. 2005).

En los masticadores de follaje se encontraron 30 morfotipos, entre los más comunes están larvas de $S$. frugiperda, Diatraea sp., M. latipes y Rupela sp. y algunos adultos de la familia Chrysomelidae. $S$. frugiperda, en particular, es reportada como una especie problemática cuando se presenta en las primeras etapas del cultivo cuando puede arrasar por completo con las plántulas (Meneses 2008). Como minador se encontró una especie común en la región: Hydrellia sp., la cual en su estado larval forma galerías en las hojas, afectando el desarrollo de la planta.

En los parasitoides se encontraron 19 morfotipos de Ichneumonidae, Braconidae y Chalcididae. Una especie afín al género Iphiaulax Foerster, 1862 (Hymenoptera: Braconidae) fue la más abundante para este grupo. Iphiaulax ha sido reportado como efectivo parasitoide de larvas de Diatraea grandiosella Diar, 1911 (Lee y Chippendale 1985).

Como observación adicional, se hallaron quistes oscuros y agujeros en el abdomen del $14 \%$ de los machos y en el 10 $\%$ de las hembras de $T$. orizicolus, que muestran evidencia de ectoparasitismo por avispas de la familia Drynidae (Fig. 5) según lo descrito por (Hernández y Belloti 1984; Mora-Kepfer y Espinoza 2009).

Riqueza y abundancia en relación con las variedades, densidades de siembra y el sistema de siembra. Al comparar las comunidades de artrópodos entre variedades de arroz no se encontraron diferencias significativas en la riqueza de especies o la abundancia de individuos (Tabla 2). En cuanto a las diferentes densidades de siembra, no se encontraron diferencias para la abundancia de artrópodos, pero si en cuanto a la riqueza, donde la densidad de $60 \mathrm{~kg} /$ ha presentó un número menor de especies con respecto a las densidades de $100 \mathrm{~kg} /$ ha y $180 \mathrm{~kg} / \mathrm{ha}$. El sistema de siembra con riego presentó una mayor abundancia total de artrópodos en comparación con el sistema secano, pero en el sistema secano hubo una mayor riqueza de especies (Tabla 2). La mayor abundancia en el sistema de riego puede explicarse por las mayores temperaturas en ese momento del año que pueden, en general, acelerar los ciclos de vida de los artrópodos (Mirhosseini et al. 2017).



Figura 2. Número de individuos de cada orden en cultivos de arroz en Yopal, Casanare. 




Figura 3. Número y porcentaje de especies por grupo funcional en cultivos de arroz en Yopal, Casanare.

\section{Dinámica poblacional de grupos funcionales y su relación} con las variables climáticas. Los cambios poblacionales de los diversos grupos funcionales representa la interacción entre los mismos. En la Tabla 3, se presenta los resultados de cómo se afecta la abundancia entre grupos. Se evidencia que las poblaciones de masticadores y chupadores está significativamente relacionadas, puesto que cualquiera de los dos grupos puede usarse como variable predictora del otro. Esto podría esperarse por ser dos grupos de herbívoros relacionados con un mismo cultivo.

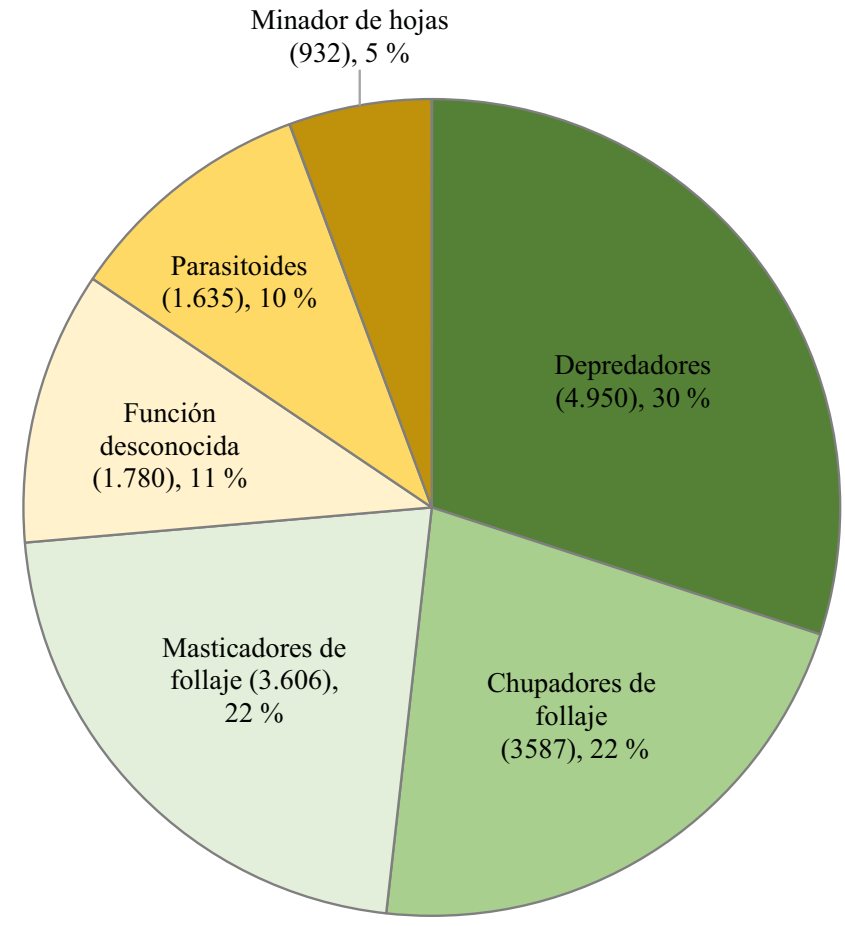

Figura 4. Número de individuos y porcentaje por grupo funcional en cultivos de arroz en Yopal, Casanare.

Por otro lado, las poblaciones de chupadores no fueron significativamente reguladas por los depredadores o los parasitoides y, teniendo en cuenta que $T$. orizicolus es el chupador más abundante en el cultivo, se podría suponer que no se presentó regulación biológica efectiva de esta plaga. Sin embargo, las poblaciones de depredadores si dependieron de los chupadores al mostrar la típica interacción depredador y presa, donde las poblaciones de depredadores aumentan con relación a la disponibilidad de alimento (Berryman 2002), pero su efecto no fué suficiente para regular las poblaciones.

Tabla 1. Morfotipos de artrópodos más abundantes en cultivos de arroz en Yopal, Casanare.

\begin{tabular}{lcc}
\hline \multicolumn{1}{c}{ Morfotipos } & $\begin{array}{c}\text { No. } \\
\text { individuos }\end{array}$ & \% \\
\hline Tetragnatha sp. (Araneae: Tetragnathidae) & 2.906 & 17,62 \\
Tagosodes orizicolus (Hemiptera: Delphacidae) & 2.078 & 12,60 \\
Especie 1 (Diptera: Syrphidae) & 1.446 & 8,77 \\
Iphiaulax sp. (Hymenoptera: Braconidae) & 1.298 & 7,87 \\
Diatraea sp. (Lepidoptera: Crambidae) & 1.100 & 6,67 \\
Spodoptera frugiperda (Lepidoptera: Noctuidae) & 1.058 & 6,41 \\
Hydrellia sp. (Diptera: Ephydridae) & 932 & 5,65 \\
Especie 2 (Orthoptera: Acrididae) & 900 & 5,46 \\
Stethorus sp. (Coleoptera: Coccinelidae) & 791 & 4,80 \\
Draeculacephala sp. (Hemiptera: Cicadellidae) & 359 & 2,18 \\
Musca sp. (Diptera: Muscidae) & 351 & 2,13 \\
Cyrtopeltis sp. (Hemiptera: Miridae) & 295 & 1,79 \\
Oebalus sp. (Hemiptera: Pentatomidae) & 172 & 1,04 \\
Amplicephalus glaucus Blanchard, 1852 (Hemiptera: Cicadellidae) & 164 & 0,99 \\
Especie 3 (Diptera: Syrphidae) & 163 & 0,99 \\
Mocis latipes (Lepidoptera: Erebidae) & 131 & 0,79 \\
Coleomegilla sp. (Coleoptera: Coccinelidae) & 116 & 0,70 \\
\hline
\end{tabular}




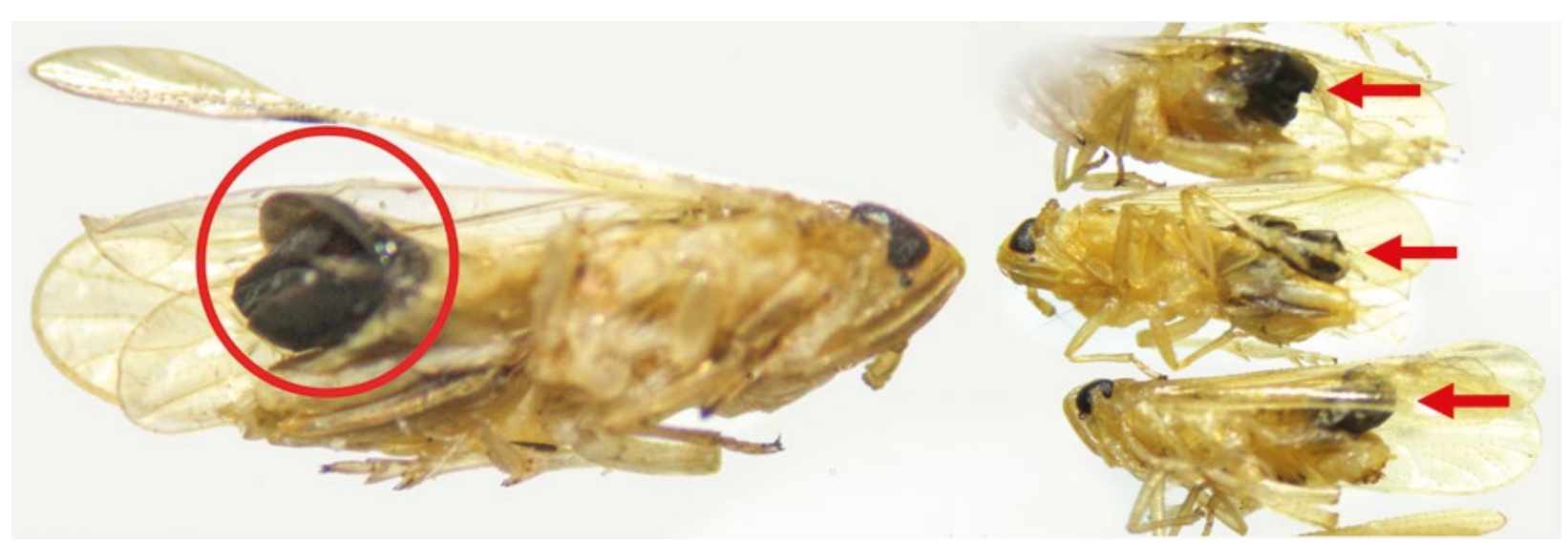

Figura 5. Tagosodes orizicolus con evidencia de ectoparasitismo en el abdomen. Las flechas rojas muestran los quistes que sobresalen del área abdominal.

Los masticadores por el contrario fueron afectados significativamente tanto por depredadores como por parasitoides, lo que indica una regulación biológica activa que valdría la pena ser explorada posteriormente para determinar las interacciones específicas entre especies. Las poblaciones de parasitoides no se vieron afectadas por ningún otro grupo.

Entre el sistema de siembra secano y sistema de riego se presentaron variaciones en la dinámica de los grupos funcionales, en relación a las fases del cultivo de arroz. En el sistema secano (Fig. 6) se observaron dos picos de masticadores durante la fase vegetativa, el primero estuvo dominado por poblaciones de $S$. frugiperda, las cuales prefieren consumir la planta en estados jóvenes, frecuentemente cortándola desde la base (Casmuz et al. 2010). En el segundo pico Diatraea sp. fue más abundante, seguida, nuevamente, por $S$. frugiperda. Este comportamiento es similar al reportado por Saavedra de Castro y Fernández Herrera (2019) quienes encontraron una fuerte asociación de $S$. frugiperda con la fase vegetativa del cultivo, que se extiende hasta parte de la fase reproductiva. El último pico poblacional de los masticadores estuvo dominado por Orthoptera, que a pesar de generar disminución del área foliar, se considera una plaga de poco impacto económico pues la planta logra compensar los daños sin afectar gravemente la producción (Meneses 2008). Sin embargo, se sugiere revisar este supuesto para las condiciones de Casanare.

Las poblaciones de los chupadores fueron siempre menores a los masticadores y su abundancia es determinada por T. oriziculus que fue la especie más común y que presentó leves fluctuaciones a lo largo del ciclo del cultivo sin darse una fuerte asociación con alguna fase fenológica en particular. Esto podría indicar el solapamiento de varias generaciones de la especie a lo largo del ciclo. Las bajas poblaciones también coinciden con lo encontrado en Venezuela por Vivas-Carmona

Tabla 2. Análisis estadístico del efecto de las variedades de arroz, densidades y sistemas de siembra sobre la riqueza y abundancia de artrópodos.

\begin{tabular}{lcccc}
\hline & \multicolumn{2}{c}{ Riqueza $(\mathbf{n = 5 2 8})$} & \multicolumn{2}{c}{ Abundancia $(\mathbf{n = 5 2 8})$} \\
\cline { 2 - 5 } & $\mathbf{t}$ & $\mathbf{P}$ & $\mathbf{t}$ & $\mathbf{P}$ \\
\hline Variedad de arroz & $-1,02$ & 0,30 & $-0,94$ & 0,34 \\
Densidad de siembra & $-2,17$ & $0,03 *$ & $-1,0$ & 0,31 \\
Sistema de siembra & $-2,8$ & $0,005 *$ & 2,5 & $0,001 *$ \\
\hline
\end{tabular}

$\mathrm{n}=$ Número de muestras; $\mathrm{t}=\mathrm{t}$ valor calculado; $\mathrm{P}=$ probabilidad de obtener valores $\geq \mathrm{t}$; *valores significativos para $\mathrm{P}<0,05$. et al. (2017), estos es, bajas poblaciones de T. oriziculus durante junio a agosto.

A partir del día 24 de la fase vegetativa, cuando las plantas se encontraban en pleno macollamiento, se observa cómo los depredadores aumentan sus poblaciones de forma similar a los masticadores brindando algún grado de control de las poblaciones de herbívoros y fluctuando con respecto a su crecimiento. Esta dinámica entre depredadores y presas ha sido observada en otras comunidades de artrópodos (Dominik et al. 2018), donde la abundancia de depredadores se correlaciona con los herbívoros del cultivo.

En el sistema con riego (Fig. 7), con excepción de los chupadores, la artropofauna se empieza a incrementar a partir del final de la fase vegetativa. Las poblaciones de depredadores y parasitoides, en particular, aumentaron durante la fase reproductiva llegando a superar las poblaciones de masticadores y chupadores lo que ofrece evidencia de su efecto en la regulación de plagas. Los depredadores más abundantes fueron arañas Tetragnathidae y para los parasitoides la familia Braconidae. En el caso de los chupadores, este grupo estuvo dominado de nuevo por T. oriziculus que presentó un comportamiento similar al sistema secano.

Las dinámicas diferenciales entre ciclos de cultivo, permiten entender cómo factores climáticos o de manejo como el riego pueden influir en las poblaciones de plagas y de controladores biológicos.

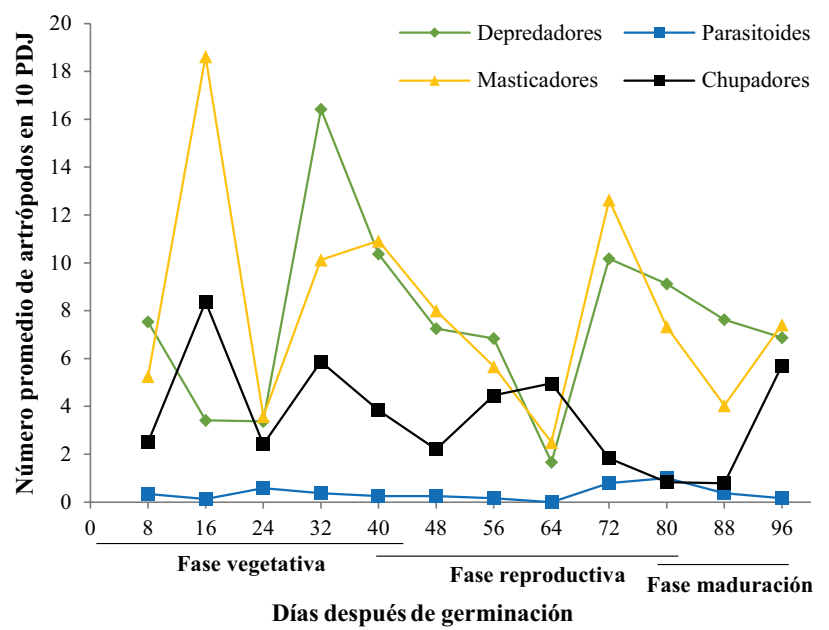

Figura 6. Promedio del número de artrópodos por grupo funcional en las diferentes etapas fenológicas del cultivo de arroz para el sistema de siembra secano. 
Tabla 3. Resultados de los modelos lineales mixtos para evaluar la interacción entre grupos funcionales de artrópodos usando como variable respuesta la abundancia de cada grupo.

\begin{tabular}{lcccccccc}
\hline & \multicolumn{2}{c}{ Chupadores } & \multicolumn{2}{c}{ Masticadores } & \multicolumn{2}{c}{ Depredadores } & \multicolumn{2}{c}{ Parasitoides } \\
\hline Abundancia & $\mathrm{t}$ & $\mathrm{P}$ & $\mathrm{t}$ & $\mathrm{P}$ & $\mathrm{t}$ & $\mathrm{P}$ & $\mathrm{t}$ & $\mathrm{P}$ \\
Chupadores & & & 2,209 & $0,027^{*}$ & 0,824 & 0,41 & 0,61 & 0,54 \\
Masticadores & 4,203 & $<0,001 *$ & & & 2,878 & $<0,001^{*}$ & 2,142 & $0,03266^{*}$ \\
Depredadores & 2,209 & $0,02765 *$ & 0,883 & 0,37786 & & & $-0,97$ & 0,33239 \\
Parasitoides & 0,767 & 0,44325 & $-0,071$ & 0,94374 & $-0,76$ & 0,86036 & & \\
\hline
\end{tabular}

$\mathrm{t}=\mathrm{t}$ valor calculado; $\mathrm{P}=$ probabilidad de obtener valores $\geq \mathrm{t}$; *valores significativos para $\mathrm{P}<0,05$.

Efecto de las variables climáticas en la dinámica poblacional. A partir del análisis Random Forest se estimó la relevancia de cada una de las variables climáticas en la abundancia de las poblaciones de artrópodos (Tabla 4). Para entender estos efectos se construyeron gráficas que muestran los puntos críticos de inflexión de las poblaciones en función de la variable climática más relevante (Figs. 8 y 9).

En la Figura 8A. se observa que a partir de $26^{\circ} \mathrm{C}$ en promedio las poblaciones de chupadores presentan una alta disminución mientras que los masticadores de follaje aumentan significativamente las poblaciones a partir de $21^{\circ} \mathrm{C}$ de temperatura mínima (Fig. 8B). Esto significa que existe un rango de temperatura entre 21 a $26^{\circ} \mathrm{C}$ que es óptimo para ambos grupos de plagas, pero a partir de $26^{\circ} \mathrm{C}$ la atención se debe centrar en los masticadores; lo cual se relaciona con el hecho de que las poblaciones de chupadores sean menores en el segundo semestre cuando la humedad relativa y las precipitaciones son significativamente reducidas en la zona y las temperaturas son mucho más altas que durante el ciclo de cultivo secano (Bustamante Lozano et al. 2013). Los depredadores también son fuertemente influenciados por la temperatura (Fig. 8C), aumentando sus poblaciones a partir de $21^{\circ} \mathrm{C}$ de temperatura mínima de forma similar a los masticadores, lo que se relaciona con las similitudes en fluctuaciones poblaciones que se presentan en estos dos grupos. Para los parasitoides (Fig. 8D), la humedad relativa fue más relevante al disminuir las poblaciones a partir de $73 \%$, lo cual se refleja en las poblaciones más abundantes en el segundo semestre cuando las condiciones son mucho más secas en Casanare (Bustamante Lozano et al. 2013).



Figura 7. Promedio del número de artrópodos por grupo funcional en las diferentes etapas fenológicas del cultivo de arroz para el sistema de siembra con riego.
En la Figura 9 se observan las variables climáticas más relevantes para las familias de artrópodos más abundantes en el cultivo. Para Tetragnathidae, el grupo de arañas más común, observamos cómo a partir de $21^{\circ} \mathrm{C}$ se marca un aumento en las poblaciones (Fig. 9A), similar al comportamiento presentado por el grupo de depredadores en su conjunto (Fig. 8C) y a la correlación positiva de temperatura y aumento en arañas encontrado en otros estudios (Masika et al. 2017). En la familia Delphacidae, donde la especie más común fue $T$. orizicolus, la variable climática más relevante es la humedad relativa, donde las poblaciones se reducen consideradamente a partir de $83 \%$ (Fig. 9B).

Para depredadores Syrphidae, la alta humedad relativa también parece ser limitante, especialmente a partir del $75 \%$, cuando las poblaciones desaparecen del cultivo (Fig. 9C). Para el caso de Ephydridae, básicamente el minador Hydrellia, la temperatura es la variable que más impacta su flujo poblacional reduciéndose a partir de $26^{\circ} \mathrm{C}$. Para los Noctuidae, en este caso representado principalmente por $S$. frugiperda, M. latipes y Diatraea, las poblaciones aumentaron a partir de $21{ }^{\circ} \mathrm{C}$ similar a lo reportado en otros estudios (Meneses 2008; Masika et al. 2017).

\section{Conclusiones}

En este estudio se hace un primer reconocimiento de la diversidad y abundancia de artrópodos asociados al cultivo de arroz en Yopal, Casanare. Se encontró que esta zona tradicionalmente arrocera, a pesar que se aplica una gran cantidad de agroquímicos, hay una población significativa de artrópodos benéficos que se debe conservar y manejar en beneficio del cultivo. En particular, se destaca la abundancia de arañas del género Tetragnatha que fue el morfotipo más común en el estudio y el principal artrópodo depredador. En cuanto a los parasitoides, avispas del género Iphiaulax en la familia Braconidae fueron las más abundantes. Dentro de los herbívoros, $T$. oriziculus fue la especie más abundante, lo que confirma que es la plaga de mayor importancia en el cultivo. $S$. frugiperda y Diatraea sp. fueron también comunes dentro de los monitoreos de artrópodos masticadores.

En cuanto a las dos variedades evaluadas (Fedearroz 174 y Fedearroz 733), no se encontraron diferencias en la riqueza o abundancia de artrópodos presentes. Así mismo, tampoco se encontraron diferencias en abundancia para las tres densidades evaluadas $(60,100$ y $180 \mathrm{~kg} / \mathrm{ha})$. Con respecto a la riqueza, esta fue significativamente menor para la densidad de $60 \mathrm{~kg} / \mathrm{ha}$ y no se encontraron diferencias entre $100 \mathrm{~kg} / \mathrm{ha}$ y $180 \mathrm{~kg} / \mathrm{ha}$.

Se encontró un efecto significativo de la abundancia de depredadores y parasitoides sobre las poblaciones de masticadores 
Tabla 4. Relevancia de las variables climáticas que contribuyen a la variación poblacional de grupos funcionales de artrópodos y algunas familias abundantes en el cultivo de arroz en Yopal, Casanare. Los valores corresponden al porcentaje de bosques y árboles en los que la variable climática fue la más discriminante en el análisis Random Forest.

\begin{tabular}{lcccccccccc}
\hline \multicolumn{1}{c}{ Grupo } & HR & T min & $\begin{array}{c}\text { T min } \\
\text { pro }\end{array}$ & T pro & T Max & RD & RS & PP. acu & $\begin{array}{c}\text { T max } \\
\text { pro }\end{array}$ & $\begin{array}{c}\text { Freq. } \\
\text { PP. }\end{array}$ \\
\hline Chupadores de follaje & 3,6 & 7,3 & 3,6 & 43,9 & 9,7 & 3,0 & 1,8 & 11,3 & 5,7 & 10,2 \\
Masticadores de follaje & 7,8 & 26,8 & 7,1 & 10,1 & 7,6 & 9,5 & 19,8 & 5,8 & 4,1 & 1,4 \\
Depredadores & 5,0 & 26,7 & 30,2 & 4,0 & 3,8 & 10,1 & 11,4 & 3,7 & 4,3 & 0,7 \\
Parasitoides & 42,4 & 3,7 & 0,3 & 8,9 & 35,9 & 0,8 & 5,8 & 2,0 & 0,2 & 0,0 \\
Araneae: Tetragnathidae & 6,7 & 26,5 & 30,6 & 2,9 & 3,7 & 12,1 & 11,0 & 2,6 & 3,2 & 0,7 \\
Hemiptera: Delphacidae & 28,4 & 8,5 & 2,8 & 6,2 & 18,3 & 14,6 & 5,3 & 4,3 & 9,7 & 2,1 \\
Diptera: Syrphidae & 61,9 & 17,9 & 1,0 & 1,5 & 7,8 & 1,3 & 1,1 & 0,2 & 7,2 & 0,1 \\
Lepidoptera: Noctuidae & 4,0 & 65,8 & 2,4 & 4,0 & 1,5 & 1,9 & 9,7 & 1,3 & 2,4 & 7,1 \\
\hline
\end{tabular}

Los valores en rojo destacan la variable más relevante. HR: Humedad relativa; T mín: Temperatura mínima; T mín pro: Temperatura mínima promedio; T pro: Temperatura promedio; T máx: Temperatura máxima; RD: Rango diurno; RS: Radiación solar; PP. acu: Precipitación acumulada; T máx pro: Temperatura máxima promedio; Frec. PP.: Frecuencia de precipitación.


Figura 8. Rango de mayor variación en la abundancia promedio de grupos de artrópodos en función de la variable climática de mayor influencia para ambos ciclos de siembra de arroz. Gráficas obtenidas a partir de la función "partialplot" en el paquete Random Forest de $\mathrm{R}$ con base en 2.000 árboles y 100 bosques. 



Figura 9. Rango de mayor variación en la abundancia promedio de familias comunes de artrópodos en función de la variable climática de mayor influencia para ambos ciclos de siembra de arroz. Gráficas obtenidas a partir de la función "partialplot" en el paquete Random Forest de R con base en 2.000 árboles y 100 bosques. 
evidenciando su rol en el control biológico de plagas. La dinámica de las poblaciones en el cultivo demostró estar altamente influenciada por las variables climáticas, siendo la humedad relativa y la temperatura las de mayor relevancia en la presencia de artrópodos. En general, se encontró, que la alta humedad relativa ( $>75-80 \%$ ) disminuye las poblaciones de artrópodos, mientras que el efecto de la temperatura varía según el grupo o la familia. En los sistemas de siembra, se encontraron diferencias en la dinámica de los artrópodos por lo que se requiere que los planes de manejo sean ajustados a las particularidades de cada ciclo.

\section{Agradecimientos}

A Alejandro Pabón, Bibiana Pérez, Nelson Madrigal, Adriana Alfonso, Frank Fierro, Irania López, Ronald Valencia y Alex Palencia por su colaboración en la recolección y conteo de los especímenes. A Fedearroz, la Universidad de la Salle y el fondo Griswold de la Universidad de Cornell. A los revisores anónimos por sus comentarios y sugerencias.

\section{Literatura citada}

BEDOYA, A.; FERNÁNDEZ HERRERA, C.; PÉREZ GARCÍA, K. D. 2018. Diversidad de la entomofauna asociada a vegetación aledaña a cultivos de arroz, maíz y algodón. Temas Agrarios 23 (2): 107-120. https://doi.org/10.21897/rta.v23i2.1295

BERRYMAN, A. 2002. Population cycles: Causes and analysis. pp. 3-28. En: Berryman, A. A. (Ed.). Population cycles: The case for trophic interactions. Oxford University Press. New York. 208 p. https://doi.org/10.1093/oso/9780195140989.003.0005

BREIMAN, L. 2001. Random forest. Machine Learning 45: 5-32. https://doi.org/10.1023/A:1010933404324

BUSTAMANTE LOZANO, Á. M.; PÁEZ MARTÍNEZ, A.; ESPITIA BARRERA, J. E.; CÁRDENAS CASTRO, E. 2013. Análisis de datos meteorológicos para identificar y definir el clima en Yopal, Casanare. Revista de Medicina Veterinaria 25: 8592. https://doi.org/10.19052/mv.2301

CASMUZ, A.; JUÁREZ, M. L.; SOCÍAS, M. G.; MURÚA, M. G.; PRIETO, S.; MEDINA, S.; WILLINK, E.; GASTAMINZA, G. 2010. Revisión de los hospederos del gusano cogollero del maíz, Spodoptera frugiperda (Lepidoptera: Noctuidae). Revista de la Sociedad de Entomología de Argentina 69 (3-4): 209-231. https://www.biotaxa.org/RSEA/article/viewFile/28610/25959

COSTA, A.; THANARAJOO, S. S.; SIVAPRAGASAM, A. 2018. Pest-Smart practices and early warning system under climate change (A manual for rice and other crops). CGIAR Research program on Climate Change, Agriculture and Food Security (CCAFS). Wageningen, Países bajos: Disponible en: https://hdl. handle.net/10568/97537 [Fecha revisión: 15 junio 2019].

CUEVAS, A. 1994. Las arañas: Controladores naturales de insectos fitófagos en el cultivo de arroz en Norte de Santander. Revista Colombiana de Entomología 20 (3): 179-185.

DEPARTAMENTO ADMINISTRATIVO NACIONAL DE ESTADÍSTICA. 2019. Encuesta nacional de arroz mecanizado (ENAM) I Semestre 2019. DANE. http://fedearroz.com.co/new/ documentos/2019/encuesta_arroz_mecanizado.pdf

DEPARTAMENTO ADMINISTRATIVO NACIONAL DE ESTADÍSTICA. 2020. Encuesta nacional de arroz mecanizado (ENAM) Semestre II 2019. DANE. https://www.dane.gov.co/ files/investigaciones/boletines/arroz/bol_arroz_IIsem19.pdf

DOMINIK, C.; SEPPELT, R.; HORGAN, F. G.; SETTELE, J.; VÁCLAVÍK, T. 2018. Landscape composition, configuration, and trophic interactions shape arthropod communities in rice agroecosystems. Journal of Applied Ecology 55 (5): 2461-2472. https://doi.org/10.1111/1365-2664.13226
FEDERACIÓN NACIONAL DE ARROCEROS. 2011. Dinámica del sector arrocero de los Llanos Orientales de Colombia 1999-2011. FEDEARROZ. http://www.fedearroz.com.co/doc_economia/ Dinamica_del_sector_arrocero_en_los_Llanos_orientales.pdf

FEDERACIÓN NACIONAL DE ARROCEROS. 2019. Estadísticas arroceras: Valoración nominal por rubros de los costos del arroz riego desde 2011 hasta 2021 Semestre 1 en Colombia. Costos por hectárea en pesos colombianos. Zona: Nacional. FEDEARROZ. Disponible en: http://www.fedearroz.com.co/ new/costos.php [Fecha revisión: 10 febrero 2020].

FERNÁNDEZ, F.; SHARKEY, M. J. (Eds.). 2006. Introducción a los Hymenoptera de la región neotropical. Sociedad Colombiana de Entomología y Universidad Nacional de Colombia. Bogotá, Colombia. 894 p.

FERNÁNDEZ, F.; ANDRADE-C., G.; AMAT, G. (Eds.). 2007. Insectos de Colombia Vol. 3. Universidad Nacional de Colombia. Bogotá, Colombia. 164 p.

HE, X.; QIAO, Y.; SIGSGAARD, L.; WU, X. 2020. The spider diversity and plant hopper control potential in the long-term organic paddy fields in sub-tropical area, China. Agriculture, Ecosystems \& Environment 295: 106921. https://doi. org/10.1016/j.agee.2020.106921

HERNÁNDEZ, M. P.; BELLOTI,A. 1984. Ciclos de vida y hábitos de Haplogonatopus hernandezae Olmi (Hymenoptera: Dryinidae) controlador natural del saltahojas del arroz Sogatodes orizicola (Muir). Revista Colombiana de Entomología 10 (3 y 4): 3-8.

LAMICHHANE, J. R.; BARZMAN, M.; BOOIJ, K.; BOONEKAMP, P.; DESNEUX, N.; HUBER, L.; KUDSK, P.; LANGRELL, S. R. H.; RATNADASS, A.; RICCI, R.; SARAH, J-L.; MESSÉAN, A. 2015. Robust cropping systems to tackle pests under climate change. A review. Agronomy for Sustainable Development 35: 443-459. https://doi.org/10.1007/s13593-014-0275-9

LEE, H. J.; CHIPPENDALE, G. M. 1985. Development of Iphiaulax kimballi (Hymenoptera: Braconidae), an ectoparasite of the southwestern corn borer, Diatraea grandiosella (Lepidoptera: Pyralidae). Journal of the Kansas Entomological Society 58 (3): 509-516. www.jstor.org/stable/25084670

LEÓN-BURGOS, A. F.; MURILLO-PACHECO, J. I.; BAUTISTA-ZAMORA, D.; QUINTO CÁNOVAS, J. 2019. Insectos benéficos asociados a plantas arvenses atrayentes en agroecosistemas del Piedemonte de la Orinoquia Colombiana. Cuadernos de Biodiversidad 56: 1-14. https://doi.org/10.14198/cdbio.2019.56.01

LOU, Y-G.; ZHANG, G-R.; ZHANG, W-Q.; HU, Y.; ZHANG, J. 2013. Biological control of rice insect pests in China. Biological Control 67 (1): 8-20. https://doi.org/10.1016/j. biocontrol.2013.06.011

MASIKA, F. B.; MASANZA, M.; ALUANA, G.; BARRIGOSSI, J. A. F.; KIZITO, E. B. 2017. Abundance, distribution and effects of temperature and humidity on arthropod fauna in different rice ecosystems in Uganda. Journal of Entomology and Zoology Studies 5 (5): 964-973. http://www.entomoljournal.com/ archives/2017/vol5issue5/PartM/5-4-120-357.pdf

MENESES, R. 2008. Manejo integrado de los principales insectos y ácaros plagas del arroz. Instituto Nacional de investigaciones del Arroz. Cuba. http://cagricola.uclv.edu.cu/descargas/libros/ LIBRO_Manejo_Integrado_de_los_principales_insectos_y_ acaros_plagas_del_arroz.pdf

MENESES, R.; REYES, L.; CALVERT, L.; TRIANA, M.; CUERVO, M.; DUQUE, M. C. 2005. Identificación de posibles biotipos de Tagosodes orizicolus en diferentes zonas arroceras de Colombia. Manejo Integrado de Plagas y Agroecología (Costa Rica) (74): 52-58. http://orton.catie.ac.cr/repdoc/A2149E/ A2149E.PDF

MIRHOSSEINI, M. A.; FATHIPOUR, Y.; REDDY, G. V. P. 2017. Arthropod development's response to temperature: a review and new software for modeling. Annals of the Entomological Society of America 110 (6): 507-520. https://doi.org/10.1093/ aesa/sax071 
MORA-KEPFER, F.; ESPINOZA, A. M. 2009. Parasitism and predation of the planthopper Tagosodes orizicolus (Homoptera: Delphacidae) by a dryinid parasitoid in Costa Rica. Revista de Biología Tropical 57 (Suppl. 1): 2003-2011. https://www.scielo.sa.cr/ scielo.php?script $=$ sci arttext\&pid $=$ S0034-77442009000500018

PEÑARANDA RINCÓN, V. H.; HIGUERA ACOSTA, O. L.; BASTIDAS, H.; HERNÁNDEZ, P.; REYES, L. A. 1999. Menejo integrado de sogata (Tagosodes orizicolus) Muir en el cultivo de arroz en los Llanos Orientales. Fedearroz-Pronatta. 15 p. http://bibliotecadigital.agronet.gov.co/bitstream/11348/6456/1/ Manejo\%20integrado\%20de\%20sogata\%20muir\%20en $\% 20$ el\%20cultivo\%20de\%20arroz.pdf

PÉREZ, C. R.; CUEVAS, A. 2012. Monitoreo de insectos y daños en el cultivo del arroz en Colombia. Revista Arroz 60 (500): 31-39. http://www.fedearroz.com.co/revistanew/arroz500.pdf

R CORE TEAM. 2019. R: A language and environment for statistical computing. R Foundation for statistical computing, Vienna, Austria. https://www.R-project.org/

RENGIFO-CORREA, L. A.; GONZÁLEZ, R. 2011. Clave ilustrada para la identificación de las familias de Pentatomomorpha (Hemiptera-Heteroptera) de distribución neotropical. Boletín Científico Centro de Museos. Museo de Historia Natural 15 (1): 168-187. http://www.scielo.org.co/scielo.php?pid=S012330682011000100014\&script $=$ sci_abstract\&tlng=es

REYNA, J.; TRABANINO, R.; AVEDILLO, M.; PITTY, A.; RUEDA, A. 1994. Inventario de plagas y algunos de sus enemigos naturales en el cultivo de arroz en Honduras. Revista CEIBA 35 (1): 35-45. Disponible en: https://bdigital.zamorano. edu/handle/11036/3687 [Fecha revisión: 28 junio 2019].

RÍOS ROMERO, R. 2003. Evaluación de la fluctuación poblacional de artrópodos en el cultivo de arroz (Oryza sativa I) Variedad capirona, bajo el sistema de trasplante en el valle del Bajo Mayo, San Martín. Universidad Nacional de San Martín. 56 p. http:// repositorio.unsm.edu.pe/handle/11458/1563

SAAVEDRA DE CASTRO, E.; FERNÁNDEZ HERRERA, C. 2019. Fluctuación poblacional de insectos fitófagos asociados a las fases fenológicas en arroz riego en dos épocas de siembra. Revista Arroz 67 (542): 16-24. http://www.fedearroz.com.co/ revistanew/arroz542.pdf

SETTLE, W. H.; ARIAWAN, H.; ASTUTI, E. T.; CAHYANA, W.; HAKIM, A. L.; HINDAYANA, D.; LESTARI, A. S. 1996.
Managing tropical rice pests through conservation of generalist natural enemies and alternative prey. Ecology 77 (7): 1975-1988. https://doi.org/10.2307/2265694

TRIPLEHORN, C. A.; JOHNSON, N. F. 2005. Borror's introduction to the study of the insects. 7 ma Edición. Thompson Brooks/Cole. Ohio, EE. UU. 864 p.

VIVAS C., L. E.; NOTZ, A. 2011. Distribución espacial en poblaciones de Oebalus insularis Stål (Hemiptera: Pentatomidae) en el cultivo de arroz en Calabozo, estado Guárico, Venezuela. Revista Científica Agrícola 11 (1): 109-125. https://dialnet.unirioja.es/ servlet/articulo? codigo $=4216182$

VIVAS-CARMONA, L. E.; ASTUDILLO-GARCÍA, D. H.; MONASTERIO-PIÑERO, P. P. 2017. Fluctuación poblacional del insecto sogata, Tagosodes orizicolus empleando una trampa de luz y su relación con variables climáticas en Calabozo Estado Guárico, Venezuela. Journal of the Selva Andina Biosphere 5 (2): 70-79. https://doi.org/10.36610/j.jsab.2017.050200070

WELCH, K. D.; HARWOOD, J. D. 2014. Temporal dynamics of natural enemy-pest interactions in a changing environment. Biological Control 75: 18-27. https://doi.org/10.1016/j. biocontrol.2014.01.004

\section{Origen y financiación}

Esta publicación es resultado del proyecto Evaluación de la fluctuación poblacional de la entomofauna asociada al cultivo de arroz, bajo las condiciones agroclimaticas de Yopal, Casanare (Proyecto: FHG-0213-PI), proyecto cofinanciado por Fedearroz y la Universidad de La Salle.

\section{Contribuciones de los autores}

Diana Obregón-Corredor y Diana Katherinne Rios-Moyano recolectaron e identificaron los especimenes.

Francisco Javier Hernández-Guzmán concibió la idea y el diseño experimental.

Diana Obregón-Corredor y Francisco Javier Hernández-Guzmán realizaron los análisis estadísticos.

Diana Obregón-Corredor elaboró el manuscrito con aportes de los otros dos autores. 